\title{
CAPÍTULO XI \\ RESPONSABILIDAD SOCIAL ESTRATEGIA \\ MERCADOLÓGICA PARA BENEFICIO SOCIAL Y DEL \\ CAPITAL INTELECTUAL EN LA INDUSTRIA TEXTILERA DE CUAUTEPEC DE HINOJOSA HIDALGO- MÉXICO
}

\section{Liliana de Jesús Gordillo Benavente}

Doctora en Dirección y Marketing, profesora investigadora de la Universidad Politécnica de Tulancingo. Correo Electrónico: liliana.gordillo@upt.edu.mx

\section{Benedicta María Domínguez Valdez}

Doctora en Dirección y Organizaciones, profesora investigadora de la Universidad Politécnica de Tulancingo. Correo Electrónico: benedicta.dominguez@upt.edu.mx

\section{Claudia Vega Hernández}

Doctorante en Gestión Administrativa, profesora investigadora de la Universidad Politécnica de Tulancingo. Correo Electrónico: claudia.vega@upt.edu.mx

\section{Anwar Alarcón Flores}

Maestro en Gestión Pública Aplicada, profesor investigador de la Universidad Politécnica de Tulancingo. Correo Electrónico: anwar.alarcon@upt.edu.mx

\section{Idania Nery Aguilar}

Maestra en Dirección de Organizaciones. Correo electrónico. nerys2022@gmail.com

\section{Resumen}

La presente investigación se realizó según el siguiente objetivo proponer la responsabilidad social como estrategia de mercadotecnia, para beneficio de la sociedad y capital intelectual en la industria textilera de Cuautepec Hinojosa- México. Metodológicamente Se desarrolló mediante un enfoque cuantitativo, de alcance descriptivo, de tipo no experimental, transeccional descriptiva, se utilizó un instrumento de investigación que midió las variables de: estrategias de responsabilidad social empresaria, ética empresarial, compromiso con la comunidad, calidad de vida del trabajador, estrategias de mercadotecnia y medio ambiente. Una vez analizada la información se encontró evidencia estadística que confirma que los textileros tienen poco conocimiento en cuanto a responsabilidad social empresarial, ya que algunos realizan estas acciones sin saber del término antes referido, otra variable que tuvo impacto fue que los empresarios textileros se preocupan por establecer valores y que son respetados en sus instalaciones y de esta manera intentan proporcionar una adecuada calidad de vida a sus trabajadores. Bajo este contexto tienen identificado variables de mercadotecnia incorporado en sus empresas, sin embargo, la participación de campañas de conciencia social y espíritu de apoyo a la sociedad es muy limitado, ya que se preocupan más por la venta de su producto. Otro resultado destacado fue que los empresarios no generan residuos que lo dañen, lo que los lleva a buscar alternativas que a su juicio no contaminan el medio que les rodea tales como buscar recicladoras de desperdicio o procesadoras de aceites, situación que favorece a estas empresas.

Palabras Claves: Responsabilidad social, industria textilera, estrategias de marketing. 


\title{
SOCIAL RESPONSIBILITY MARKET STRATEGY FOR SOCIAL AND INTELLECTUAL CAPITAL BENEFIT IN THE TEXTILLE INDUSTRY OF CUAUTEPEC DE HINOJOSA HIDALGO- MÉXICO
}

\begin{abstract}
Abstrac
The present investigation was carried out according to the following objective to propose social responsibility as a marketing strategy, for the benefit of society and intellectual capital in the textile industry of Cuautepec Hinojosa. Methodologically It was developed by means of a quantitative approach, of descriptive scope, non-experimental type, descriptive transectional, a research instrument was used that measured the variables of; strategies of corporate social responsibility, business ethics, commitment to the community, quality of life of the worker, marketing strategies and the environment. Once the information was analyzed, statistical evidence was found confirming that the textile workers have little knowledge in terms of corporate social responsibility, since some carry out these actions without knowing the aforementioned term, another variable that had an impact was that textile entrepreneurs worry about establish values and that are respected in their facilities and in this way they try to provide an adequate quality of life to their workers. In this context they have identified marketing variables incorporated in their companies, however, the participation of campaigns of social awareness and spirit of support for society is very limited, since they are more concerned with the sale of their product. Another outstanding result was that businessmen do not generate waste that harms it, which leads them to look for alternatives that in their opinion do not pollute the environment that surrounds them, such as looking for waste recyclers or oil processors, a situation that favors these companies.
\end{abstract}

Keywords: Social responsibility, textile industry, marketing strategies

\section{Capítulo resultado de un proyecto de investigación culminado}

\section{Introducción}

En este mundo globalizado donde cada día se hace más evidente que la empresa al formar parte de la sociedad se convierte en punto central a observar y es esa sociedad el juez que decide si una empresa u organización se comporta de forma empáticamente responsable o no. La presente investigación, centra su eje motriz en el conocimiento y oportuna aplicación de la Responsabilidad Social Empresarial en la industria textilera de la región de Cuautepec de Hinojosa; esto derivado de que la región es eminentemente textilera; por lo que se precisa poder proponer la responsabilidad social como estrategia de mercadotecnia, para beneficio de la sociedad y capital intelectual en la industria textilera de Cuautepec Hinojosa.

De acuerdo a Argandoña (2012) en una investigación realizada sostiene que una de las principales problemáticas presentadas en las industrias textilera de Cuautepec Hinojosa 
Hidalgo, es el desconocimiento del término de responsabilidad social su significado y lo que podría aportar a la empresa, a los empleados y la sociedad en general, una referencia a tres ámbitos de responsabilidad, económico, social y medioambiental. Sin embargo, no existe un registro en la entidad que aporte información al respecto y por ende la aplicabilidad de la misma.

El objetivo de la investigación es proponer la responsabilidad social como estrategia de mercadotecnia, para beneficio de la sociedad y capital intelectual en la industria textilera de Cuautepec Hinojosa- México. Es conveniente realizar esta investigación, ya que servirá para conocer la idiosincrasia de los empresarios textileros y la carente importancia que dan a brindar apoyo a la sociedad y al trabajador en un apoyo conjunto determinado responsabilidad social.

De tal manera se dará a conocer al sector textil los elementos que forman la responsabilidad social. Así mismo tendrá relevancia social entre la comunidad textilera en general, ya que al conocer lo que es la responsabilidad social su trascendencia en la sociedad generará un lazo de compromiso entre la empresa y la sociedad, así como la empatía entre la sociedad y las diferentes empresas, mismos que redundarán en mejoras en el entorno y por ende en las empresas y empleados de tal forma que poco a poco la Responsabilidad Social Empresaria (RSE) será parte del día a día en estas empresas, situación que la sociedad cuautepequense notaría en cambios en el ambiente y educación social de los empleados de las empresas que lo practiquen.

Las variables que se estudiaron en esta investigación son: Estrategias de Responsabilidad Social Empresarial, Ética empresarial, Compromiso con la comunidad, Calidad de vida del trabajador, Estrategias de mercadotecnia y medio ambiente.

Las etapas planteadas por los autores y que se llevaron a cabo en esta investigación fueron: planteamiento del problema, justificación, objetivo general, revisión de literatura, diseño de la metodología de la investigación, resultados y conclusiones, es así como cada una de las etapas propiciará el desarrollo de la investigación haciendo saber que la responsabilidad social funge como estrategia mercadológica para beneficio de la sociedad así como del capital intelectual en las industrias textileras.

\section{Fundamentación teórica}

\section{Mercadotecnia}

De acuerdo a Kotler y Keller (2012), el marketing trata de identificar y satisfacer las necesidades humanas y sociales. Una de las mejores y más cortas definiciones de marketing es "satisfacer las necesidades de manera rentable" p.90. En este orden de ideas consiste en buscar la satisfacción de las necesidades, deseos y expectativas de los consumidores mediante un grupo de actividades coordinadas que, al mismo tiempo, permita a la organización alcanzar sus metas. La satisfacción de los clientes es lo más importante, para lograrlo, la organización debe investigar cuáles son las necesidades, deseos y expectativas del cliente para poder crear verdaderos satisfactores (Fisher y Espejo, 2011). Si bien es cierto el marketing juega un papel muy importante para todas las organizaciones, ya que busca la satisfacción de las necesidades, deseos y expectativas del cliente, así como la generación de estrategias, logrará que las empresas generen ventajas competitivas. 


\section{Responsabilidad Social Empresarial}

La responsabilidad social es obligación de un individuo de manera particular, o puede ser tarea de un grupo de individuos que conforman una empresa, una organización etc., que va dirigida a la adopción de tareas, estrategias, líneas de acción, etc. así como al cumplimiento de éstas en aras de lograr una armonía social, empresarial, gubernamental o familiar que contribuyen a la disminución de los impactos negativos que las acciones del hombre ocasionan en la sociedad, el medio ambiente o en las propias relaciones sociales. (Velez y Cano, 2016).

Por su parte, Capriotti y Shulze (2010), señalan que en la actualidad un número creciente de compañías contribuyen al diseño, mantenimiento y desarrollo del entorno social, por medio de sus políticas y actividades de responsabilidad social empresarial (RSE). Es en este sentido que las empresas no son ya observadas sólo como actores económicos, sino que también han pasado a ser (obligados por la fuerza de los hechos) actores sociales activos, en una sociedad que busca resolver las cuestiones de equilibrio entre el nuevo orden económico y el orden social, en busca de un reparto equitativo. La RSE impacta en la reputación de la empresa, por tanto en la evaluación global que los stakeholders (clientes y público en general) tienen de una compañía a lo largo del tiempo. Esta evaluación está basada en sus experiencias directas con la organización y en cualquier otra información directa o indirecta sobre la misma, en comparación con las experiencias e informaciones de otras compañías (la competencia incluída). En este orden de ideas el tema de RSE se ha convertido en nuestros días en un tema fundamental para todas las empresas, y por ende debe de considerarse como una estrategia mercadológica en pro de la sociedad.

\section{Capital Intelectual}

De acuerdo a Brooking (2001), en el término capital intelectual se hace referencia a la combinación de activos intangibles que generan capacidades distintivas o competencias esenciales a largo plazo y que permiten funcionar a la empresa. Por su parte Bernárdez, (2008) menciona que el capital intelectual puede ser definido en consecuencia como aquellos productos, procesos o capacidades generados por la actividad espiritual e intelectual humana se transmiten como cultura. De la misma forma capital intelectual se define como el conjunto de activos intangibles de una organización que, pese a no estar reflejados en los estados contables tradicionales, en la actualidad generan valor o tiene potencial de generarlo en el futuro (Carrión, 2007). Si bien es cierto el capital intelectual es el pilar más preciado que tiene la organización y se le debe de prestar la mejor atención, ya que éstos son los que propiciarán el éxito organizacional.

\section{Metodología}

Esta investigación tiene un enfoque cuantitativo, permitió medir las variables para determinar el grado en qué los empresarios entienden que es responsabilidad social y si la aplican en sus organizaciones. Así mismo el diseño de la investigación fue de tipo no experimental, transeccional, descriptiva, ya que los estudios solo se observan los fenómenos 
en su ambiente natural para analizarlos y transeccional descriptivo, ya que indagan los hechos de las modalidades, categorías o niveles de una o más variables en una población, son estudios puramente descriptivos. Hernández, Fernandez y Baptista (2014), es decir que se recolectan los datos de un solo momento, en un tiempo único. La recopilación de esta información dará sustento a la técnica de investigación cuantitativa.

La población de estudio de las empresas textileras de la región de Cuautepec son de 200 empresarios. Se procedió a realizar la muestra con la aplicación de la fórmula estadística para muestras finitas referida por Arias (2012), y dio como resultado de 132 sujetos de investigación. El estudio empleó muestras probabilísticas, por lo cual se consideró cualquier empresa de la región que realice actividades textiles.

Se realizó un instrumento de investigación con preguntas abiertas y afirmaciones en escala de Likert que es una escala psicométrica utilizada principalmente en la investigación de mercados para la comprensión de las opiniones y actitudes de un consumidor hacia una marca, producto o mercado meta. El instrumento evaluó 22 afirmaciones con escala de Likert, y 8 preguntas abiertas además de 5 preguntas como datos de identificación. Para medir la validez y confiabilidad del instrumento se llevó a cabo mediante el Alfa de Cronbach y de acuerdo a la tabla 1 , se visualiza el alfa con .820 de la cual se observó que el instrumento fue confiable.

Tabla 1. Alfa de Crombach

\begin{tabular}{cc}
\hline \multicolumn{2}{c}{ Estadísticos de fiabilidad } \\
\hline Alfa de Cronbach & $\mathrm{N}$ de elementos \\
\hline .820 & 22 \\
\hline
\end{tabular}

Fuente: Elaboración propia (2019).

Para el procesamiento y análisis de datos se midieron los resultados a través del programa estadístico Statistical Package for the Social Sciences (SPSS), en el cual se introdujeron los datos para analizar e interpretar cada una de las variables de forma descriptiva, que permitirá el cumplimiento del objetivo general de la investigación.

\section{Resultados}

Los resultados de la investigación parten del objetivo general que fue proponer a la responsabilidad social como estrategia de mercadotecnia para la industria textilera de Cuautepec de Hinojosa, para la mejora hacia la sociedad y capital intelectual. 


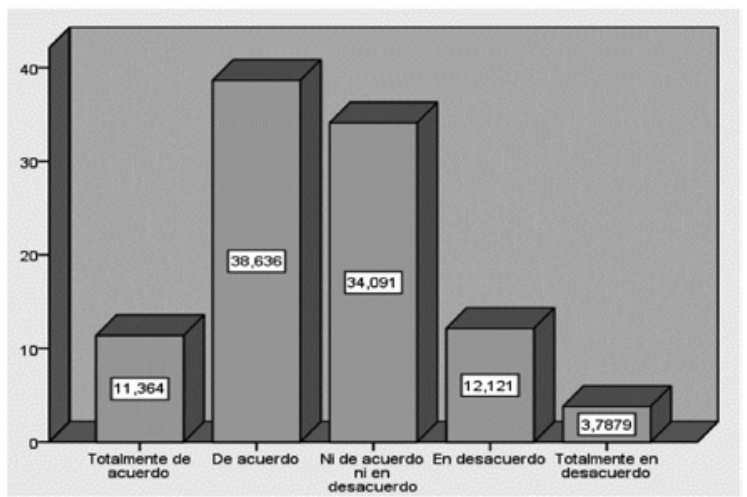

\section{Gráfica 1. Conocimiento del termino Responsabilidad Social}

Fuente: Elaboración propia (2019).

La gráfica 1, muestra el conocimiento del término de Responsabilidad Social en donde, el $38.6 \%$ opinaron estar de acuerdo de que conocen de lo que esta expresión significa, mientras que $34.1 \%$ de los encuestados son indiferentes al respecto lo que significa, que no están seguros de saber con precisión lo que este término significa. Por otro lado, un $12.1 \%$ estuvieron en desacuerdo lo cual significa que no saben lo que es la Responsabilidad Social mientras en otro extremo $11.4 \%$ dijeron estar totalmente de acuerdo lo que nos habla de conocimiento específico respecto al significado del término. Finalmente $3.8 \%$ lo que refiere a 5 de las personas encuestadas mencionaron no conocer absolutamente nada de este término. Bajo esta estadística se puede afirmar que un 50\% conoce lo que significa el término de la responsabilidad social, aunado esto es necesario que el 100\% de los encuestados conozcan que la RS es una obligación que deben de tener las organizaciones para el bienestar social.

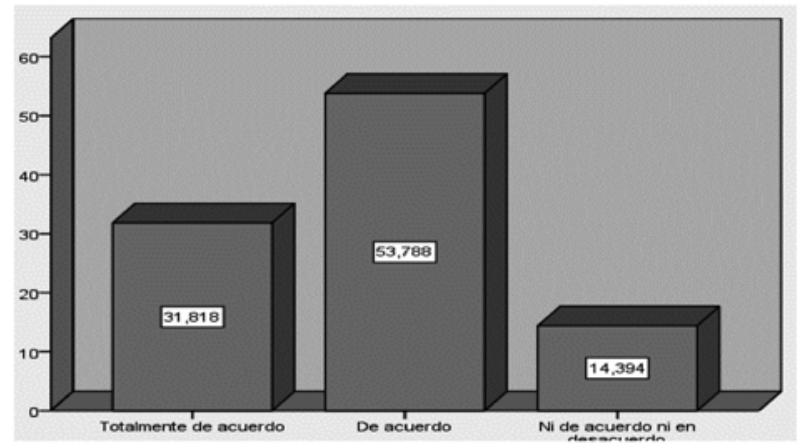

Gráfica 2. Valores

Fuente: Elaboración propia (2019). A partir de los datos obtenidos del SPSS 
De acuerdo a la gráfica 2 , se visualiza sobre los valores que se practican en la empresa por el que el 53.8\% están de acuerdo con la idónea aplicación de valores, mientras el 31.8\% están totalmente de acuerdo, en tanto solo $14.4 \%$ son indiferentes a este cuestionamiento dado que no tienen certeza de aplicarlos aunque mencionan si tenerlos establecidos. Esto nos lleva a determinar que el $100 \%$ de las empresas textileras de la región de Cuautepec, aplican algún valor dentro de la empresa por lo que para las organizaciones es un elemento esencial en término de Responsabilidad Social.

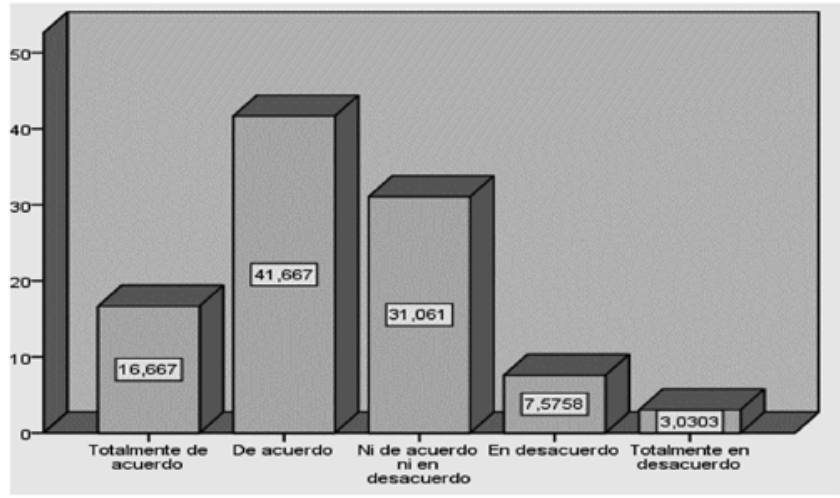

\section{Gráfica 3. Necesidades comunitarias}

Fuente: Elaboración propia (2019).

La gráfica número 3 muestra que si los textileros identifican las necesidades que tiene su comunidad el $41.7 \%$ están de acuerdo con la aseveración, mientras que el $31.1 \%$ están ni de acuerdo ni en desacuerdo, en tanto que el $16.7 \%$ están totalmente de acuerdo. Por lo que se puede deducir que es un aspecto muy importante dentro del término de responsabilidad social identificar las necesidades dentro de la comunidad, ya que de acuerdo a la literatura este término viene encaminado a las líneas de acción para lograr los impactos negativos que el hombre ocasiona en la sociedad, de ahí que los empresarios deben de estar pendientes en las necesidades de su comunidad para contribuir al desarrollo social. 


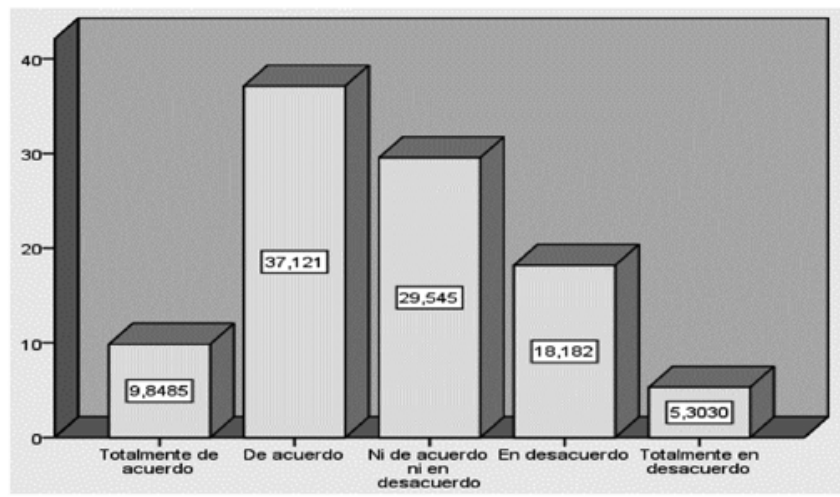

\section{Gráfica 4. Mejoramiento de las condiciones de vida}

Fuente: Elaboración propia (2019).

La gráfica 4 muestra la participación en el mejoramiento de las condiciones de vida de la comunidad el $37.1 \%$ están de acuerdo con esta situación, el $29.5 \%$ ni de acuerdo ni en desacuerdo, mientras que el $18.2 \%$ está en desacuerdo; un $9.8 \%$ están totalmente de acuerdo finalmente un $5.3 \%$ están totalmente en desacuerdo. Así se puede conjuntar que el $47 \%$ de los textileros apoyan en el mejoramiento de sus comunidades, un $29 \%$ le resulta indiferente este apoyo, mientras que al $23.5 \%$ personas no apoyan en nada a su comunidad. De ahí se desprende que más del 50\% no participa para el mejoramiento de las condiciones de vida de su comunidad, relacionado a que ellos mismos no conocen el término de responsabilidad social, lo que permite la concientización de los empresarios para que participen con el término de responsabilidad social aplicado.

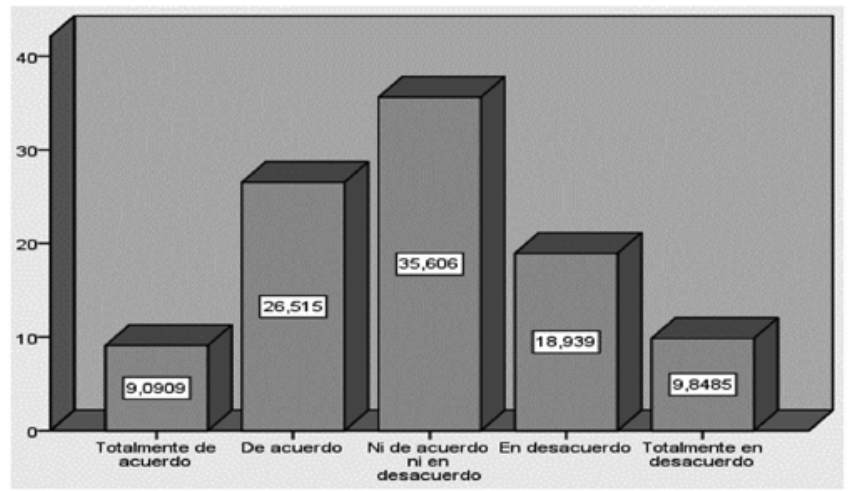

\section{Gráfica 5. Soluciones de la comunidad}

Fuente: Elaboración propia (2019). 
En este orden de ideas en la gráfica 5 muestra la aportación a las soluciones a la comunidad el $35.6 \%$ están ni de acuerdo ni en desacuerdo, mientras el $26.5 \%$ están de acuerdo, en tanto el $18.9 \%$ están en desacuerdo, por otro lado $9.8 \%$ están totalmente en desacuerdo, mientras $9.1 \%$ equivalente están totalmente de acuerdo. De esto deriva que los empresarios textileros brindan soluciones en una escala baja, ya que un $35.6 \%$ brindan algún tipo de soluciones, así mismo y en igual porcentaje $35.6 \%$ se manifiestan indiferente,s en ocasiones apoyan o no, sin generar ningún cambio en el ambiente. Finalmente, un $28.7 \%$ no aportan ningún tipo de solución.

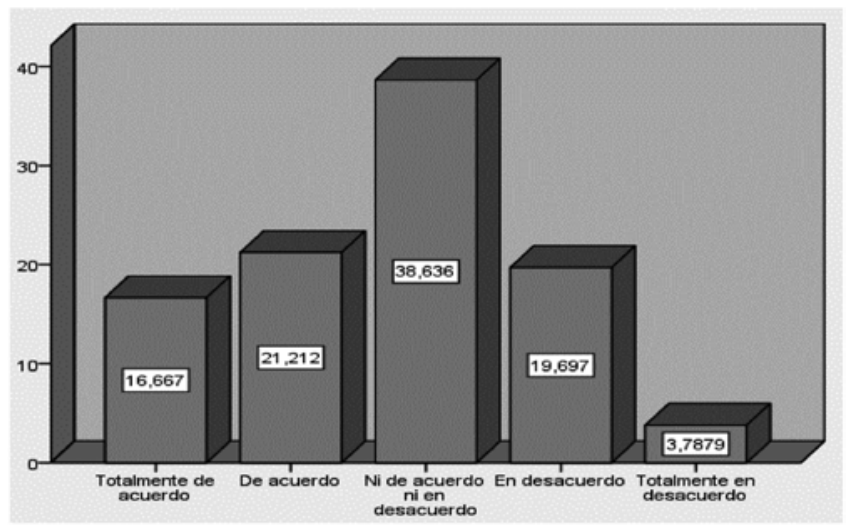

Gráfica 6. Reconocimiento de la marca

Fuente: Elaboración propia (2019).

Al hablar del conocimiento que se tiene de la marca en la región el 38.6\% no están ni de acuerdo ni en desacuerdo, mientras el $21.2 \%$ están de acuerdo, no obstante el $19.7 \%$ están en desacuerdo, mientras un $16.7 \%$ están totalmente de acuerdo, finalmente un $3.8 \%$ o 5 personas comentaron estar totalmente en desacuerdo, tal como se muestra en la gráfica 6. Por lo anterior se puede decir que $38.6 \%$ mencionan que su marca no es conocida ni desconocida en la región mientras un 37.9\% dijeron que su marca si es conocida en la región, sin embargo el $23.5 \%$ revelaron que su marca no es conocida en la región de Cuautepec. 


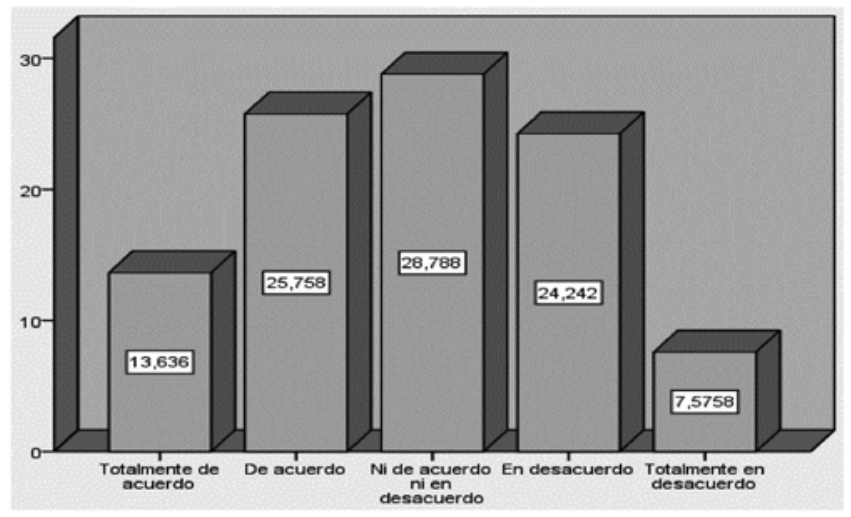

\section{Gráfica 7. Misión, visión y valores}

Fuente: Elaboración propia (2019).

Al preguntar respecto a si la misión, visión y valores son identificadas por todos los miembros de la empresa el $28.8 \%$ mencionaron no estar ni de acuerdo ni en desacuerdo, un $25.8 \%$ están de acuerdo, mientras un $24.2 \%$ están en desacuerdo, un $13.6 \%$ están totalmente de acuerdo para finalmente un $7.6 \%$ están totalmente en desacuerdo, tal como aparece en la gráfica 7. Por lo que un aspecto importante de la responsabilidad social es el manejo de la misión, visión y valores del conjunto total que integran la empresa, por lo que se tiene que reforzar esta parte en las textileras.

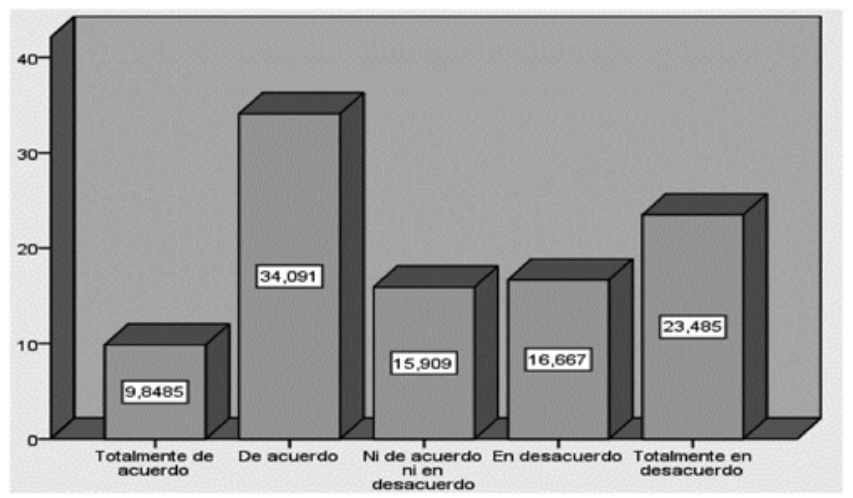

\section{Gráfica 8. Campañas de conciencia social}

Fuente: Elaboración propia (2019).

Respecto a si han participado en campañas de conciencia social el $34.1 \%$ están de acuerdo, mientras un $2.5 \%$ están totalmente en desacuerdo, un $16.7 \%$ están en desacuerdo, 
así un $15.9 \%$ comentó no estar ni de acuerdo ni en desacuerdo y un $9.8 \%$ están totalmente de acuerdo. Gráfica 8. Por lo anterior se puede establecer que un $49.3 \%$ refirieron haber participado en alguna campaña de conciencia social, mientras un 40.2\% manifestaron no haber participado en ninguna campaña de conciencia social, finalmente un $15.9 \%$ no manifestaron con claridad su participación o ausencia en este tipo de campañas. Si bien es cierto este es un factor importante en el término de responsabilidad social, por lo que más del $50 \%$ no han participado en campañas social, que contribuye a que no están aplicando la responsabilidad social en sus empresas, y por ende se debe de concientizar participar en campañas que contribuyan al mejoramiento del desarrollo social.

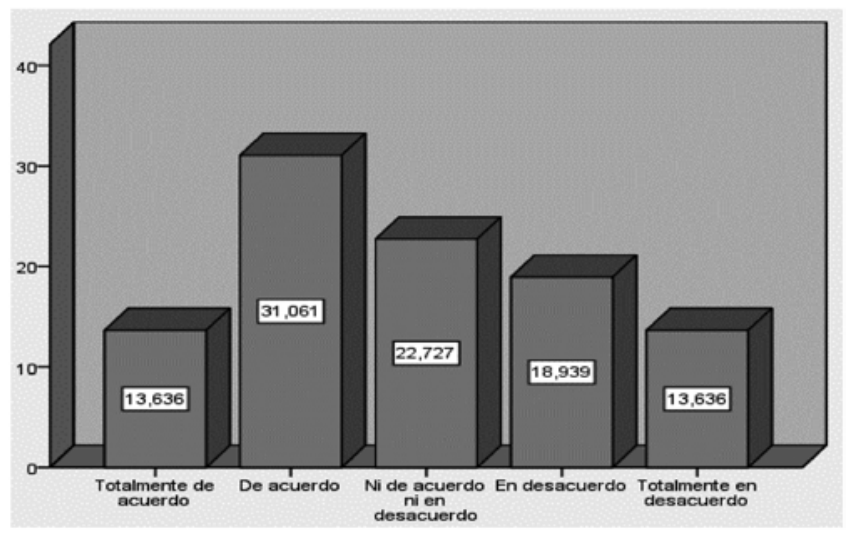

\section{Gráfica 9. Espíritu de apoyo a la sociedad}

Fuente: Elaboración propia (2019).

Al preguntar respecto a si se fomenta el espíritu de apoyo a la sociedad el $31.1 \%$ están de acuerdo, el $22.7 \%$ no están ni de acuerdo ni en desacuerdo, un $18.9 \%$ están en desacuerdo, mientras paralelamente $13.6 \%$ expresaron estar totalmente de acuerdo en igualdad con otro $13.6 \%$ están totalmente en desacuerdo. Lo que lleva a concluir que realmente las empresas textileras en cuestión no fomentan la responsabilidad social, ya que no se ve apoyo en la sociedad, y esto viene a propiciar a que se tienen que establecer estrategias que permita a las organizaciones lograr un bien en pro de la sociedad. 


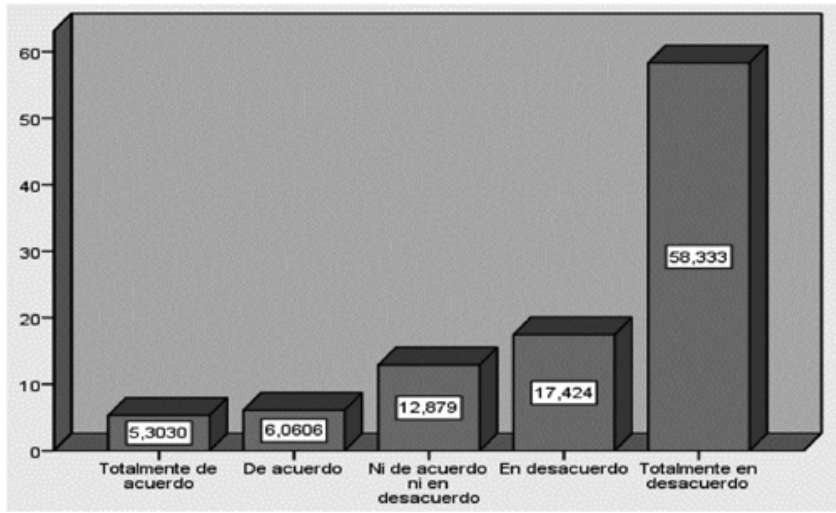

\section{Gráfica 10. Generación de residuos}

Fuente: Elaboración propia (2019).

La gráfica 10, hace referencia sobre la generación de residuos, es decir que si la empresa genera residuos que dañen al medio ambiente un 58.3\% manifestó totalmente en desacuerdo y un $17.4 \%$ expresaron estar en desacuerdo, mientras un $12.9 \%$ se pronunció ni de acuerdo ni en desacuerdo, lo que dejo a un $6.1 \%$ opinaron estar de acuerdo y un $5.3 \%$ están totalmente de acuerdo. De lo anterior se puede visualizar que más del $50 \%$ de las empresas textileras no generan residuos por lo que cumplen un factor de la responsabilidad social que de acuerdo a la teoría de Velez y Cano (2016) comentan que se deben disminuir los impactos negativos ocasionados en la sociedad y medio ambiente por lo que las empresas contribuyen con este factor.

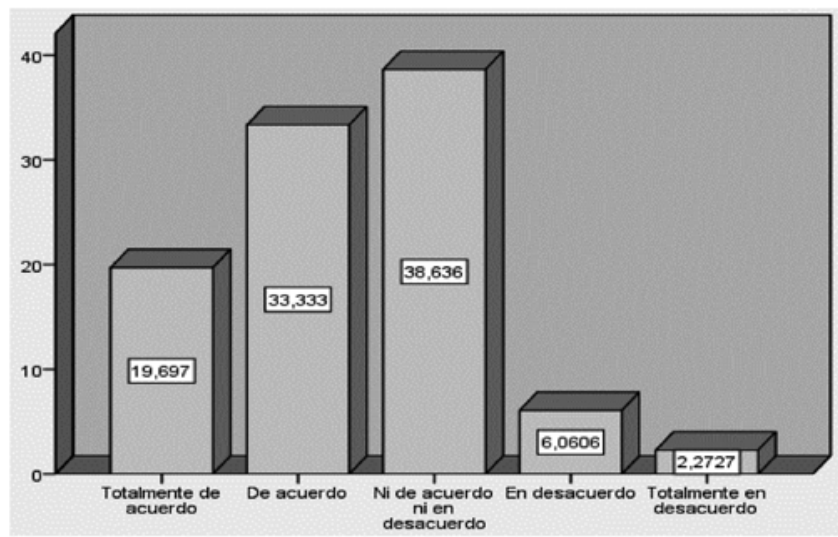

Gráfica 11. Medio ambiente en la localidad

Fuente: Elaboración propia (2019). 
De acuerdo a las necesidades del medio ambiente en su localidad un 38.6\% comentó no estar ni de acuerdo ni en desacuerdo, lo cual genera una indolencia por parte de los empresarios al no prestar atención a las necesidades del medio que los cobija, mientras un 33.3\% expresó estar de acuerdo lo cual habla de una compenetración con el medio que los rodea, un $19.7 \%$ están totalmente de acuerdo lo que muestra una plena cercanía de la empresa y la comunidad, un $6.1 \%$ están en desacuerdo lo que manifiesta poca importancia de los empresarios a la comunidad en la que se desenvuelven y un $2.3 \%$ están totalmente en desacuerdo. Como se puede observar el $40 \%$ de los empresarios no tienen una cultura para cuidar el medio ambiente lo que es una consecuencia negativa para la sociedad, ya que muchas organizaciones no cumplen con la responsabilidad social.

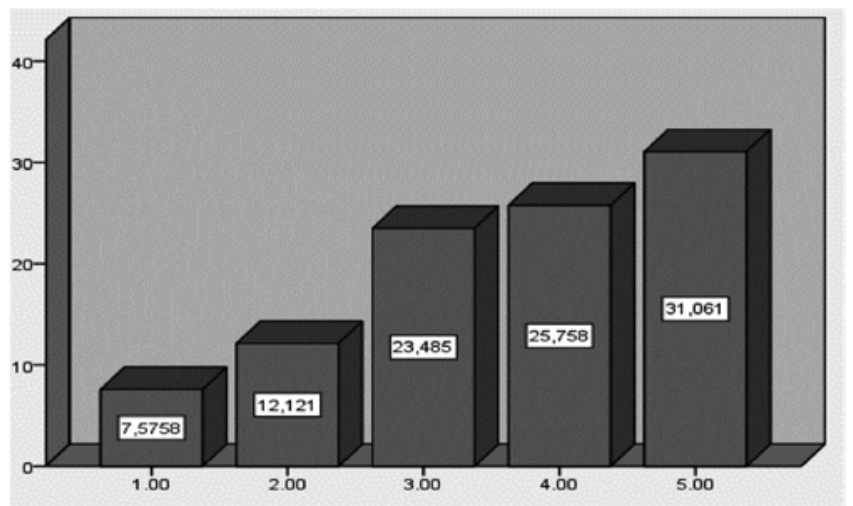

\section{Gráfica 12. Residuos del medio ambiente}

Fuente: Elaboración propia (2019).

De acuerdo a la gráfica 12, se visualiza si han evaluado sus residuos en el medio ambiente un $31.1 \%$ expresó estar totalmente en desacuerdo, un $25.8 \%$ están en desacuerdo, un $23.5 \%$ se pronunció ni de acuerdo ni en desacuerdo, mientras $12.1 \%$ refirieron estar de acuerdo, finalmente un $7.6 \%$ comentaron estar totalmente de acuerdo. De lo anterior se puede puntualizar que $56.9 \%$ no han realizado una evaluación del impacto que generan sus residuos en el medio ambiente, por lo que de acuerdo a la literatura se debe de fomentar entre los empresarios textileros de Cuautepec la concientización para que las empresas sean socialmente responsables, en pro de la sociedad y capital intelectual.

\section{Conclusiones}

Una vez analizados los resultados obtenidos por la investigación, se puede concluir que los empresarios textileros de la región de Cuautepec están divididos en cuanto a conocimiento del termino Responsabilidad Social Empresarial, ya que mientras algunos ejercen acciones con conciencia de lo que conlleva esta actividad, existe quien realiza 
habitualmente actividades sin conocer que son acciones propias de la Responsabilidad Social Empresarial, esto finalmente trae un impacto muy fuerte en la sociedad, ya que si no se tienen la concientización de los daños que se generan al no corresponder con la responsabilidad social, muy pronto esta situación se estará convirtiendo en un problema radical en toda la población y por ende la contaminación ambiental, por tal razón se debe fomentar acciones que permitan lograr que las empresas fomente la responsabilidad social.

En este tenor la sociedad Textilera de Cuautepec, no tiene claras las necesidades que existen en su comunidad, motivo por el cual no resulta relevante brindar soluciones o apoyar a la entidad en cuanto a condiciones de vida, derivado de lo anterior solo un $38.6 \%$ de los textileros identifica su responsabilidad con futuras generaciones; sin embargo el $100 \%$ de los empresarios textiles se preocupa por establecer valores que sean respetados en sus instalaciones, de esta manera intentan generar una adecuada calidad de vida para sus trabajadores, situación que no todos consigue, solo el $44 \%$ de los empresarios brindan capacitación continua y son quienes realmente se comprometen con sus empleados más allá de pagar el sueldo establecido. Por otro lado, si bien un $83.3 \%$ de los Textileros refieren contar con un logotipo y un $50 \%$ hablo de una frase que los identifica, su participación de campañas de conciencia social y el espíritu de apoyo a la sociedad es muy divido y restringido dado que la única preocupación existente es su producto, sin darse cuenta de la importancia que conlleva la sociedad que les rodea.

No obstante, consideran importante el medio ambiente motivo por el cual un $75.7 \%$ de los empresarios intentan no generar residuos que lo dañen, lo que los lleva a buscar alternativas que a su juicio no contaminan el medio que les rodea tales como buscar recicladoras de desperdicio o procesadoras de aceites; empero solo un $19.7 \%$ han realizado una verdadera evaluación del impacto de sus residuos en el medio ambiente, ya que en realidad desconocen ese factor.

Se hace necesario con esta investigación dar una aportación a los empresarios textileros de la Cuautepec Hinojosa Hidalgo, para concientizarlo sobre su actuación con ética, respetando al medio ambiente, a la sociedad y a su capital intelectual y por ende a sus clientes, debido a que si actúan de una forma con responsabilidad social contribuyen al mejoramiento del medio ambiente.

\section{Referencias Bibliográficas}

Aguilera, C.A.; Puerto, B.D., (2012). Crecimiento empresarial basado en la Responsabilidad Social. Pensamiento \& Gestión, núm. 32, pp. 1-26

Arias, Fidias G., (2012). El proyecto de investigación, Introducción a la metodología cientifica. Episteme. Caracas Venezuela.

Argadoña Antonio, (2012) Otra definición de responsabilidad social, IESE Business School pp 2

Barroso T., (2008). La responsabilidad social empresarial. Un estudio en cuarenta empresas de la ciudad de Mérida, Yucatán. Contaduria y administración, no.226. 
Bernárdez, M.L., (2008). Capital intelectual Creación de valor en la sociedad del conocimiento. Estados Unidos de América: AuthorHouse

Brooking Annie. (1997). El capital intelectual. El principal activo de las empresas del tercer milenio. Paidós. Barcelona

Carrión, M.J., (2007). Estrategia, de la visión a la acción. España: ESIC

Capriotti Peri Paul y Shukze Lepe Francisca. (2010). Responsabilidad Social Empresarial. Libros de la Empresa Executive Business Scholl. España

Dopico P., Rodríguez D., González V., (2012). La responsabilidad social empresarial y los stakeholders: un análisis clúster. Revista Galega de Economía, vol. 21, núm. 1, pp. 1-17

Hernández Sampieri Roberto, Fernandez Collado Carlos y Baptista Lucio María del Pilar. (2010) Metodología de la investigación. Mc.GrawHill Interamericana editores. México

Kotler Philip y Lane Keller Kevin. (2012). Dirección de Marketing. Decimocuarta edición Pearson Education, México.

Fisher Laura y Espejo Jorge. (2011) Mercadotecnia. Mc. Graw Hill Education. México.

Mercado S., García H., (2007). La responsabilidad social en empresas del valle de Toluca (México). Un estudio exploratorio. Estudios Gerenciales, vol.23, no.102.

Vélez-romero y Cano-Lara Evelin D, Los diferentes tipos de responsabilidad social y sus implicaciones éticas. The different types of social responsibility and their ethical implications. Diferentes tipos de responsabilidad social. Ciencias Sociales y Políticas ISSN: 2477-8818 Vol. 2, núm. esp., dic., 2016, pp. 117-12 